\title{
Neutrophil-to-Lymphocyte Ratio is a Prognostic Predictive Factor for Local Advanced Lower Rectal Cancer Treated with Curative Surgical Treatment after Neoadjuvant Chemoradiotherapy: A Single- Center Retrospective Cohort Study
}

\section{Ryosuke Nakagawa}

Tokyo Women's Medical University: Tokyo Joshi lka Daigaku

Shimpei Ogawa ( $\nabla$ ogawa.shimpei@twmu.ac.jp )

Tokyo Women's Medical University: Tokyo Joshi lka Daigaku https://orcid.org/0000-0001-6428-1209

Yuji Inoue

Tokyo Women's Medical University: Tokyo Joshi lka Daigaku

\section{Takeshi Ohki}

Tokyo Women's Medical University: Tokyo Joshi lka Daigaku

Yoshiko Bamba

Tokyo Women's Medical University: Tokyo Joshi Ika Daigaku

Kurodo Koshino

Tokyo Women's Medical University: Tokyo Joshi lka Daigaku

Kimitaka Tani

Tokyo Women's Medical University: Tokyo Joshi Ika Daigaku

Hisako Aihara

Tokyo Women's Medical University: Tokyo Joshi Ika Daigaku

Michio Itabashi

Tokyo Women's Medical University: Tokyo Joshi lka Daigaku

Masakazu Yamamoto

Tokyo Women's Medical University: Tokyo Joshi Ika Daigaku

Research article

Keywords: Neutrophil-to-lymphocyte ratio, relapse-free survival, rectal cancer, neoadjuvant chemoradiotherapy

Posted Date: April 5th, 2021

DOl: https://doi.org/10.21203/rs.3.rs-386980/v1 
License: (c) (i) This work is licensed under a Creative Commons Attribution 4.0 International License. Read Full License 


\section{Abstract}

Background: The neutrophil-to-lymphocyte ratio (NLR) correlates with relapse-free survival (RFS) and may be a predictor of recurrence in patients after curative surgery for colorectal cancer. This study aimed to analyze the long-term oncological outcomes of locally advanced lower rectal cancer treated with curative surgery after neoadjuvant chemoradiotherapy (nCRT) to examine the prognostic value of the NLR and to evaluate the fluctuation of pre- and post-CRT NLR as recurrence risk factors.

Methods: Fifty-two patients who underwent curative surgery were enrolled between 2009 and 2016. A cutoff pre-CRT NLR of 3.20 was used based on receiver-operating characteristic curve analysis. The primary outcome was RFS. Factors influencing recurrence after treatment according to fluctuations between the pre- and post-CRT NLR were also analyzed.

Results: Univariate analysis was performed using 17 clinicopathological factors thought to affect RFS. A significant difference was found in the pre-CRT NLR (hazard ratio [HR]: 7.626, 95\% confidence interval [CI]: 2.760-21.06, $p<0.0001$ ), operation time (HR: 2.949, 95\% Cl: 1.137-7.646, $p=0.0261$ ), and pathological T stage (HR: 8.342, 95\% Cl: 2.458-28.306 p=0.0007). RFS according to the pre-CRT NLR using KaplanMeier analysis showed that the group with pre-CRT $\geq 3.20$ had a lower 5 -year RFS $(p=0.001)$. A lower preCRT NLR resulted in a significantly higher recurrence rate, regardless of the increase or decrease in the pre- and post-CRT NLR.

Conclusions: The pre-CRT NLR may be a predictor of prognosis in patients with locally advanced lower rectal cancer after nCRT.

\section{Background}

Colorectal cancer (CRC) is one of the most common cancers of the digestive organs worldwide. In recent years, the mortality rate of patients with CRC has been steadily improving due to advancements in surgical techniques and multidisciplinary treatment. Tumor node metastasis (TNM) and Dukes classification have long been used as prognostic factors and can be used to predict the therapeutic effect when selecting the appropriate treatment for individual patients [1] [2] [3]. However, it is expected that new biomarkers will be found [4] [5] The neutrophil-to-lymphocyte ratio (NLR), which is an index of inflammation and immune function, correlates with the host inflammatory response and cancer growth $[6,7]$ and has been used as a prognostic factor in various types of carcinoma [8]. The NLR may be a defining factor, with a high NLR suggested to indicate a poor prognosis [9-11]. Neutrophils play an important role in the acidity of ligands that induce tumor cell proliferation and the invasion and cytokines that induce angiogenesis; therefore, it is considered that the increase in neutrophils in patients with CRC promotes tumor growth and metastasis[12]. Lymphocytes control the immune function of the host, and a decrease in lymphocytes impairs the host's antitumor immunity, thus affecting prognosis [13]. Therefore, the balance between neutrophils and lymphocytes is reported to reflect the tumor-promoting environment and antitumor immunity [14] I The NLR may correlate with relapse-free survival (RFS) in patients with 
stage II and stage III CRC and may affect prognosis [15]. In Europe and the United States, neoadjuvant chemoradiotherapy (nCRT) has already been reported as a standard treatment because local control is beneficial for locally advanced rectal cancer and contributes to the prognosis $[16,17]$. T cells, which are lymphocytes in tumors, are well known as prognostic factors in various solid tumors [18]. The NLR has potential as a determinant of therapeutic effect and as a prognostic predictor [19] [20] [8] [21].

We previously compared nCRT using intermittent oral tegafur-uracil (UFT) plus leucovorin (LV) to surgery alone, evaluating the efficacy, toxicity, and 3-year outcomes in our retrospective study of Japanese patients at a single center; the 3-year OS and DFS rates were $92 \%$ and $78 \%$, respectively. nCRT with intermittent oral UFT plus LV caused low toxicity, facilitated a high completion rate of CRT among patients, and provided a better local control rate [22]. Here, we investigated the correlation between RFS and the NLR and its influence on the recurrence rate of fluctuations between the pre- and post-CRT NLR in these nCRT cases.

\section{Methods}

\section{Study participants}

Fifty-two out of 63 patients underwent nCRT for lower rectal cancer from 2009 to 2016 (excluding cStage IV and double cancer but including metastatic lateral lymph node metastasis cases from cStage IV). These patients with rectal cancer at clinical tumor stages (cT) 3-4 or clinical node stages (cN) 1-2 underwent curative surgery at the Department of Gastroenterology and General Surgery, Tokyo Women's Medical University. Since 2008, we have been performing nCRT at this institution in patients with rectal cancer at $\mathrm{CT}$ stages 3-4 or $\mathrm{CN}$ stages 1-2 to prevent postoperative local recurrence. All patients were administered nCRT with intermittent oral UFT plus LV. We collected full blood count data from blood samples obtained prior to the initiation of $\mathrm{nCRT}$ and just before surgery. The NLR was calculated by dividing the absolute neutrophil count by the absolute lymphocyte count.

We evaluated the patient characteristics, clinicopathological findings, prognoses, and pre-CRT NLR. Every patient was classified according to the Union for International Cancer Control (UICC) 7th edition TNM staging system. Although CRC with metastasis to lateral lymph nodes is defined as stage IV in the UICC TNM staging system, total mesolectal excision (TME) plus lateral lymph node resection has become the standard surgical treatment for stage II and stage III primary lower rectal cancer according to the Japanese Society for Cancer of the Colon and Rectum guidelines [23]. Therefore, we included surgical resection cases with lateral lymph node dissection in this study.

All participants provided written informed consent before the study commenced.

The study protocol was approved by the Institutional Review Board of Tokyo Women's Medical University, Tokyo, Japan.

Research was conducted in accordance with the principles of the 1964 Declaration of Helsinki and its later amendments. Imaging analysis 
Computed tomography (CT) or magnetic resonance imaging was used to evaluate tumor infiltration and the presence of lymph nodes (e.g., para-rectal lymph nodes, lateral pelvic lymph nodes [LLNs]) $>1 \mathrm{~cm}$ in diameter in the shortest dimension as lymph node metastasis-positive or lymph nodes with clinical characteristics suggestive of metastasis.

\section{Chemoradiotherapy}

An overview of the nCRT protocol performed at our institution is illustrated in Fig. 1, as previously reported [22]. The oral UFT plus LV regimen consisted of two cycles, every 3 weeks. The dose of UFT was $300 \mathrm{mg} / \mathrm{m}^{2} /$ day, and the dose of LV was $75 \mathrm{mg} /$ day on days $1-14$. Chemotherapy was intermittently repeated every 28 days, followed by a 2-week break. Radiotherapy (RT) consisted of 45 Gy delivered in fractions of $1.8 \mathrm{~Gy} /$ day on weekdays for 5 consecutive weeks concomitantly with chemotherapy. RT was administered with a linear particle accelerator (Clinac 21EX; Varian Medical Systems, Inc., Palo Alto, California, USA), and radiation treatment planning was completed using the Eclipse TM system (version 7.3.10; Varian Medical Systems, Inc.). A radiotherapist determined the treatment plan based on the CT results. Preoperative RT (total dose of $45 \mathrm{~Gy}$ ) was delivered as photons with a 10-MV linear accelerator in 25 fractions ( $1.8 \mathrm{~Gy} /$ day; 5 fractions per week). RT was delivered to the pelvis through individually shaped portals, using a 3-field or 4-field box technique (anterior field, posterior field, and left and right lateral fields). Patients were monitored through an interview, physical examination, and blood tests every 2 weeks.

\section{Surgical Procedures}

Surgery was performed $\geq 8$ weeks after the completion of nCRT. Patients underwent curative operations for lower rectal cancer. Cases of non-curative resection were excluded from our study. The surgical procedure involved a pelvic lateral lymph node dissection (LLND) using a standard TME technique. At our institution, LLND is performed only for curative treatment; therefore, $\operatorname{CLNN(+)}$ was performed on the basis of preoperative size, irrespective of whether the size of LLN decreased after nCRT.

\section{Statistical Analyses}

The optimal NLR cutoff values for RFS were determined using receiver-operating characteristic (ROC) analysis. Cox proportional hazard regression analysis was performed for chi-square, univariate, and multivariate comparisons. The rates of RFS were determined using the Kaplan-Meier method. Comparisons between survival curves were performed using the log-rank test. The pre-CRT NLR was divided into two groups, a "High NLR" group and "Low NLR" group, with a cutoff value of 3.20 set as the boundary. Furthermore, the NLRs of pre-and post-CRT were compared, and those with an increase in postCRT NLR were described as "up" and those with a decrease in post-CRT NLR were described as "down." These combinations were defined as four groups ("Low NLR-down," “Low NLR-up," "High NLR-down," 
"High NLR-up"). Statistical analyses were conducted using JMP Pro 15 (SAS Institute Inc., Cary, NC, USA); $p$-values $<0.05$ were considered statistically significant.

\section{Results}

\section{Patients and tumor characteristics}

Table 1 summarizes the characteristics of the 52 patients. The median age of the study population was 64 (42-84) years, and male sex was predominant. About $96 \%$ of patients had clinical T3 or T4 stage and about $77 \%$ of patients had clinical lymph node metastasis positive $(\mathrm{cN}+)$ status. The median value of the pre-CRT NLR was 2.55 (1.11-8.63). In this population, the TME procedure rate was $52 \%$ and the abdominoperineal resection rate was $48 \%$. All patients underwent curative surgery. The pathological complete response (CR) rate was $8 \%$. Distant recurrence after surgical treatment occurred in $35 \%$ of patients, whereas local recurrence occurred in $6 \%$ of patients.

\section{Optimal Pre-crt NIr Cut Off Value For Rfs}

The optimal NLR cutoff value for RFS was determined using ROC analysis. A pre-CRT NLR of 3.20 was determined as the optimal cutoff. The area under the curve was 0.648 (Fig. 2).

\section{Univariate And Multivariate Analysis Factors With Rfs}

Univariate analysis was performed using 17 clinicopathological factors that are thought to affect RFS. The pre-CRT NLR, operation time, and pathological T stage (ypT) showed significant differences ( $p<$ 0.05). When a multivariate analysis was performed using these three factors, a significant difference was found in the pre-CRT NLR (HR: 7.626 (95\% Cl: 2.760-21.06); $p<0.0001)$, operation time (HR: 2.949 (95\% Cl: 1.137-7.646); $p=0.0261)$, and ypT (HR: 8.342 (95\% Cl: 2.458-28.306); $p=0.0007)$ (Table 2).

\section{Correlation Between The Pre-crt NIr And Rfs}

RFS according to the pre-CRT NLR was assessed using Kaplan-Meier analysis. The "High NLR" group (with pre-CRT $\geq 3.20$ ) showed a lower 5 -year RFS ( $p=0.001$ ) (Fig. 3). Comparisons of the increase and decrease in the NLR between pre-CRT and post-CRT among these four groups are shown in Fig. 4 . The distant recurrence rate tended to be particularly high in the "Low NLR-up" group (17.3\%) and the "High NLR-up" group (15.4\%). In addition, the local recurrence rate tended to be particularly high in the "High NLR-down" group (17.3\%) (Table 3). RFS according to these four groups was assessed using KaplanMeier analysis. In particular, both the "High NLR-up" and "High NLR-down" group showed a lower 5-year RFS ( $p=0.04)$ (Fig. 5). 


\section{Discussion}

Our findings suggest that the pre-CRT NLR may be a predictor of prognosis in patients with locally advanced lower rectal cancer after nCRT. The NLR reflects the balance of host immunity between pro- and anti-tumor activities. A high circulating neutrophil count is considered to have pro-tumor properties because inflammatory cytokines released from neutrophils induce angiogenesis and promote tumor growth [26].

Various cutoff values have been used to define elevated NLR in previous studies. Although some studies used cutoff values calculated by statistical methods, the reported NLR cutoff values on patients with rectal cancer who underwent nCRT are inconsistent [27-30]. In this study, a cut-off value of 3.20 for the pre-CRT NLR was significantly related to RFS. Although the pre-CRT NLR score, operation time, and ypT were significantly associated with RFS in the univariate analyses, multivariate Cox proportional hazard regression analysis showed that the pre-CRT NLR was independently associated with RFS. This demonstrates that a pre-CRT NLR greater than 3.20 is a predictor of poor prognosis. "High NLR" suggests a pro-tumor status and a higher possibility of poor radiation response, while "Low NLR" suggests an antitumor status and a higher possibility of a positive tumor response and survival [20] [21, 31] [32]. Chiang et al. showed that preoperative NLR $>3$ was associated with a larger tumor size, elevated CEA, and an increased risk of cancer progression and decreased survival in a retrospective analysis including 3,857 patients with stage I to III CRC; interestingly, no significant associations were found in the rectal cancer subgroup [33]. In this study, although all patients with advanced lower rectal cancer underwent nCRT, the group with pre-CRT NLR $\geq 3.20$ showed a lower 5-year RFS than the group with pre-CRT NLR $<3.20$. This association might be the reason why, as we previously reported, a significant difference was observed in the estimated 3-year local recurrence rate between the group who underwent nCRT and the surgery only group [22].

To our knowledge, one critical component of the tumor microenvironment is the immune system, and it has become increasingly evident that the immune system plays an integral role in preventing and promoting the development of cancer [34]. Lymphocytes, especially CD4 + or CD8 + T cells and B cells, are thought to play a key role in antitumor host immunity by inducing tumor cell apoptosis [24]. A number of immune cells, in addition to lymphocytes, can directly and indirectly kill cancer cells. In fact, immune cells patrol the body, monitoring all altered cells that become cancerous in a process known as "immunosurveillance." Through immunosurveillance, the body can effectively recognize and eliminate cancerous cells prior to them causing harm. It is already widely known that lymphocytes play an important role in anticancer activity. Patients with a low NLR have more lymphocytes relative to neutrophils in their blood than patients with a high NLR. This may alter the effectiveness of anti-cancer effects, which ultimately resulted in better RFS in the "High NLR" group of this study (Fig. 3).

In this study, we calculated post-CRT NLR in addition to pre-CRT NLR and examined whether it could be a recurrence risk factor by comparing the pre- and post-before and post-CRT NLR (Table 3). According to the results, distant recurrence tended to occur more frequently in the "High NLR-up" group. In addition, 
distant recurrence tended to occur more often in the "Low NLR-up" group, which means that the post-CRT NLR was higher than pre-CRT NLR. This may suggest that lower proportions of lymphocytes that make up NLR may be a risk of distant recurrence because of reduced "immunosurveillance."

The setting of the cutoff value by the ROC curve in medical treatment is used to determine how much sensitivity should be pursued for the benefit of the patient; however, there is no rule as to what point should be pursued. It is important to constantly re-evaluate the interpretation of the numerical values, taking into consideration the purpose of utilization, the characteristics of the target population, the degree of progression of the stage, and the effects of the development of therapeutic means. Information that can be easily obtained from blood tests is important for advanced rectal cancer, where multidisciplinary treatment is becoming mainstream. The NLR can be measured at any facility at a low cost and can be measured frequently in daily medical care. It has a utility value not found in markers and is considered to have great clinical applicability.

In this study, we used the NLR to evaluate the prognosis of local advanced rectal cancer after nCRT; however, in recent years, several other reports have evaluated it using the plate-to-lymphocyte ratio (PLR) [25] [35] and the monocyte-to-lymphocyte ratio (MLR)[36] [37] [38]. There is no consensus on which ratio is best to use, including the NLR, and it is hoped that more clinically applicable and simple biomarkers will be discovered.

A limitation of this study is that the sample size was small and provided insufficient data to draw confirmative results, due to the retrospective study design. A future well-controlled, large-scale prospective study is needed to further validate the study findings.

\section{Conclusions}

Our study suggests that an elevated NLR is a negative predictive marker and may be an independent prognostic marker for RFS in patients with local advanced rectal cancer after nCRT.

\section{Abbreviations}

NLR

Neutrophil-to-lymphocyte ratio

RFS

Relapse-free survival

nCRT

Neoadjuvant chemoradiotherapy

$\mathrm{HR}$

Hazard ratio

$\mathrm{Cl}$

Confidence interval 
CRC

Colorectal cancer

TNM

Tumor node metastasis

UFT

Tegafur-uracil

LV

Leucovorin

cT

clinical tumor stages

$\mathrm{CN}$

clinical node stages

TME

Total mesolectal excision

CT

Computed tomography

LLLNs

Lateral pelvic lymph nodes

RT

Radiotherapy

LLND

Lateral lymph node dissection

ROC

Receiver-operating characteristic

$\mathrm{cN}+$

clinical lymph node metastasis positive

$\mathrm{CR}$

Complete response

ypT

pathological T stage

PLR

Plate-to-lymphocyte ratio

MLR

Monocyte-to-lymphocyte ratio

\section{Declarations}

Ethics approval and consent to participate:

The Institutional Review Board of Tokyo Women's Medical University approved by the study (approval number 5265). This study was conducted based on the ethical guidelines of the Declaration of Helsinki. 
Informed consent was obtained from all individual participants included in this study.

\section{Consent for publication:}

Informed consent was obtained from all individual participants included in this study.

\section{Availability of data and material:}

Please contact the corresponding author with requests for data.

\section{Competing interests:}

The authors of this manuscript have no relevant conflicts of interest or financial disclosures to report.

\section{Funding:}

Not applicable.

\section{Authors' contributions:}

Writing-review and editing: all authors.

\section{Acknowledgements:}

The authors wish to thank the Department of Radiation Oncology, Tokyo Women's Medical University (Tokyo, Japan) for support the radiotherapy.

\section{References}

1. Hashiguchi Y, Hase K, Kotake K, Ueno H, Shinto E, Mochizuki H, Yamamoto J, Sugihara K. Evaluation of the seventh edition of the tumour, node, metastasis (TNM) classification for colon cancer in two nationwide registries of the United States and Japan. Colorectal Dis. 2012;14(9):1065-74.

2. Jass JR, Morson BC. Reporting colorectal cancer. J Clin Pathol. 1987;40(9):1016-23.

3. Anders A, Dressler S, Kourias E, Knauf P. [TNM-classification in surgery of colon]. Langenbecks Arch Chir. 1972;331(2):158-68.

4. Li MX, Liu XM, Zhang XF, Zhang JF, Wang WL, Zhu Y, Dong J, Cheng JW, Liu ZW, Ma L, et al.

Prognostic role of neutrophil-to-lymphocyte ratio in colorectal cancer: a systematic review and metaanalysis. Int J Cancer. 2014;134(10):2403-13.

5. Mei Z, Liu Y, Liu C, Cui A, Liang Z, Wang G, Peng H, Cui L, Li C. Tumour-infiltrating inflammation and prognosis in colorectal cancer: systematic review and meta-analysis. $\mathrm{Br} \mathrm{J}$ Cancer. 2014;110(6):1595-605.

6. Coussens LM, Werb Z. Inflammation and cancer. Nature. 2002;420(6917):860-7. 
7. Balkwill F, Mantovani A. Inflammation and cancer: back to Virchow? Lancet. 2001;357(9255):53945.

8. Templeton AJ, McNamara MG, Seruga B, Vera-Badillo FE, Aneja P, Ocana A, Leibowitz-Amit R, Sonpavde G, Knox JJ, Tran B, et al. Prognostic role of neutrophil-to-lymphocyte ratio in solid tumors: a systematic review and meta-analysis. J Natl Cancer Inst. 2014;106(6):dju124.

9. Yamanaka T, Matsumoto S, Teramukai S, Ishiwata R, Nagai Y, Fukushima M. The baseline ratio of neutrophils to lymphocytes is associated with patient prognosis in advanced gastric cancer. Oncology. 2007;73(3-4):215-20.

10. Sarraf KM, Belcher E, Raevsky E, Nicholson AG, Goldstraw P, Lim E. Neutrophil/lymphocyte ratio and its association with survival after complete resection in non-small cell lung cancer. $J$ Thorac Cardiovasc Surg. 2009;137(2):425-8.

11. Garcea G, Ladwa N, Neal CP, Metcalfe MS, Dennison AR, Berry DP. Preoperative neutrophil-tolymphocyte ratio (NLR) is associated with reduced disease-free survival following curative resection of pancreatic adenocarcinoma. World J Surg. 2011;35(4):868-72.

12. Terzić J, Grivennikov S, Karin E, Karin M. Inflammation and colon cancer. Gastroenterology. 2010;138(6):2101-14.e2105.

13. Lin EY, Pollard JW. Role of infiltrated leucocytes in tumour growth and spread. Br J Cancer. 2004;90(11):2053-8.

14. An X, Ding PR, Li YH, Wang FH, Shi YX, Wang ZQ, He YJ, Xu RH, Jiang WQ. Elevated neutrophil to lymphocyte ratio predicts survival in advanced pancreatic cancer. Biomarkers. 2010;15(6):516-22.

15. Kubo T, Ono S, Ueno H, Shinto E, Yamamoto J, Hase K. Impact of the perioperative neutrophil-tolymphocyte ratio on the long-term survival following an elective resection of colorectal carcinoma. Int J Colorectal Dis. 2014;29(9):1091-9.

16. Sauer R, Becker H, Hohenberger W, Rodel C, Wittekind C, Fietkau R, Martus P, Tschmelitsch J, Hager E, Hess $\mathrm{CF}$, et al. Preoperative versus postoperative chemoradiotherapy for rectal cancer. N Engl J Med. 2004;351(17):1731-40.

17. Park IJ, You YN, Agarwal A, Skibber JM, Rodriguez-Bigas MA, Eng C, Feig BW, Das P, Krishnan S, Crane $\mathrm{CH}$, et al. Neoadjuvant treatment response as an early response indicator for patients with rectal cancer. J Clin Oncol. 2012;30(15):1770-6.

18. Sato E, Olson SH, Ahn J, Bundy B, Nishikawa H, Qian F, Jungbluth AA, Frosina D, Gnjatic S, Ambrosone $\mathrm{C}$, et al. Intraepithelial CD8 + tumor-infiltrating lymphocytes and a high CD8+/regulatory $T$ cell ratio are associated with favorable prognosis in ovarian cancer. Proc Natl Acad Sci U S A. 2005;102(51):18538-43.

19. Malietzis G, Giacometti M, Kennedy RH, Athanasiou T, Aziz O, Jenkins JT. The emerging role of neutrophil to lymphocyte ratio in determining colorectal cancer treatment outcomes: a systematic review and meta-analysis. Ann Surg Oncol. 2014;21(12):3938-46.

20. Kim IY, You SH, Kim YW. Neutrophil-lymphocyte ratio predicts pathologic tumor response and survival after preoperative chemoradiation for rectal cancer. BMC Surg. 2014;14:94. 
21. Carruthers R, Tho LM, Brown J, Kakumanu S, McCartney E, McDonald AC. Systemic inflammatory response is a predictor of outcome in patients undergoing preoperative chemoradiation for locally advanced rectal cancer. Colorectal Dis. 2012;14(10):e701-7.

22. Nakagawa R, Inoue $Y$, Ohki T, Kaneko Y, Maeda F, Yamamoto M. Efficacy and short-term outcomes of preoperative chemoradiotherapy with intermittent oral tegafur-uracil plus leucovorin in Japanese rectal cancer patients: a single center experience retrospective analysis. World J Surg Oncol. 2017;15(1):112.

23. Watanabe T, Itabashi M, Shimada Y, Tanaka S, Ito Y, Ajioka Y, Hamaguchi T, Hyodo I, Igarashi M, Ishida H, et al. Japanese Society for Cancer of the Colon and Rectum (JSCCR) Guidelines 2014 for treatment of colorectal cancer. Int J Clin Oncol. 2015;20(2):207-39.

24. Badoual C, Hans S, Rodriguez J, Peyrard S, Klein C, Agueznay Nel H, Mosseri V, Laccourreye O, Bruneval P, Fridman WH, et al. Prognostic value of tumor-infiltrating CD4 + T-cell subpopulations in head and neck cancers. Clin Cancer Res. 2006;12(2):465-72.

25. Abdallah EA, Souza ESV, Braun AC, Gasparini VA, Kupper BEC, Tariki MS, Tarazona JGR, Takahashi RM, Aguiar Junior S, Chinen LTD. A higher platelet-to-lymphocyte ratio is prevalent in the presence of circulating tumor microemboli and is a potential prognostic factor for non-metastatic colon cancer. Transl Oncol. 2021;14(1):100932.

26. McCourt M, Wang JH, Sookhai S, Redmond HP. Proinflammatory mediators stimulate neutrophildirected angiogenesis. Arch Surg. 1999;134(12):1325-31. discussion 1331 - 1322.

27. Shen L, Zhang H, Liang L, Li G, Fan M, Wu Y, Zhu J, Zhang Z. Baseline neutrophil-lymphocyte ratio $(>/=2.8)$ as a prognostic factor for patients with locally advanced rectal cancer undergoing neoadjuvant chemoradiation. Radiat Oncol. 2014;9:295.

28. Jung SW, Park IJ, Oh SH, Yeom SS, Lee JL, Yoon YS, Kim CW, Lim SB, Lee JB, Yu CS, et al. Association of immunologic markers from complete blood counts with the response to preoperative chemoradiotherapy and prognosis in locally advanced rectal cancer. Oncotarget. 2017;8(35):5975765.

29. Shen J, Zhu Y, Wu W, Zhang L, Ju H, Fan Y, Zhu Y, Luo J, Liu P, Zhou N, et al. Prognostic Role of Neutrophil-to-Lymphocyte Ratio in Locally Advanced Rectal Cancer Treated with Neoadjuvant Chemoradiotherapy. Med Sci Monit. 2017;23:315-24.

30. Jeon BH, Shin US, Moon SM, Choi JI, Kim MS, Kim KH, Sung SJ. Neutrophil to Lymphocyte Ratio: A Predictive Marker for Treatment Outcomes in Patients With Rectal Cancer Who Underwent Neoadjuvant Chemoradiation Followed by Surgery. Ann Coloproctol. 2019;35(2):100-6.

31. Kitayama J, Yasuda K, Kawai K, Sunami E, Nagawa H. Circulating lymphocyte number has a positive association with tumor response in neoadjuvant chemoradiotherapy for advanced rectal cancer. Radiat Oncol. 2010;5:47.

32. Krauthamer M, Rouvinov K, Ariad S, Man S, Walfish S, Pinsk I, Sztarker I, Charkovsky T, Lavrenkov K. A study of inflammation-based predictors of tumor response to neoadjuvant chemoradiotherapy for locally advanced rectal cancer. Oncology. 2013;85(1):27-32. 
33. Chiang SF, Hung HY, Tang R, Changchien CR, Chen JS, You YT, Chiang JM, Lin JR. Can neutrophil-tolymphocyte ratio predict the survival of colorectal cancer patients who have received curative surgery electively? Int J Colorectal Dis. 2012;27(10):1347-57.

34. Markman JL, Shiao SL. Impact of the immune system and immunotherapy in colorectal cancer. J Gastrointest Oncol. 2015;6(2):208-23.

35. Lu C, Gao P, Yang Y, Chen X, Wang L, Yu D, Song Y, Xu Q, Wang Z. Prognostic evaluation of platelet to lymphocyte ratio in patients with colorectal cancer. Oncotarget. 2017;8(49):86287-95.

36. Basile D, Garattini SK, Corvaja C, Montico M, Cortiula F, Pelizzari G, Gerratana L, Audisio M, Lisanti C, Fanotto V, et al. The MIMIC Study: Prognostic Role and Cutoff Definition of Monocyte-to-Lymphocyte Ratio and Lactate Dehydrogenase Levels in Metastatic Colorectal Cancer. Oncologist. 2020;25(8):661-8.

37. Lisanti C, Basile D, Parnofiello A, Bertoli E, Andreotti VJ, Garattini SK, Bartoletti M, Cattaneo M, Di Nardo $P$, Bonotto $M$, et al. The SENECA study: Prognostic role of serum biomarkers in older patients with metastatic colorectal cancer. J Geriatr Oncol. 2020;11(8):1268-73.

38. Jakubowska K, Koda M, Grudzinska M, Kanczuga-Koda L, Famulski W. Monocyte-to-lymphocyte ratio as a prognostic factor in peripheral whole blood samples of colorectal cancer patients. World $\mathrm{J}$ Gastroenterol. 2020;26(31):4639-55.

\section{Tables}

Due to technical limitations, table 1 to 3 is only available as a download in the Supplemental Files section.

\section{Figures}

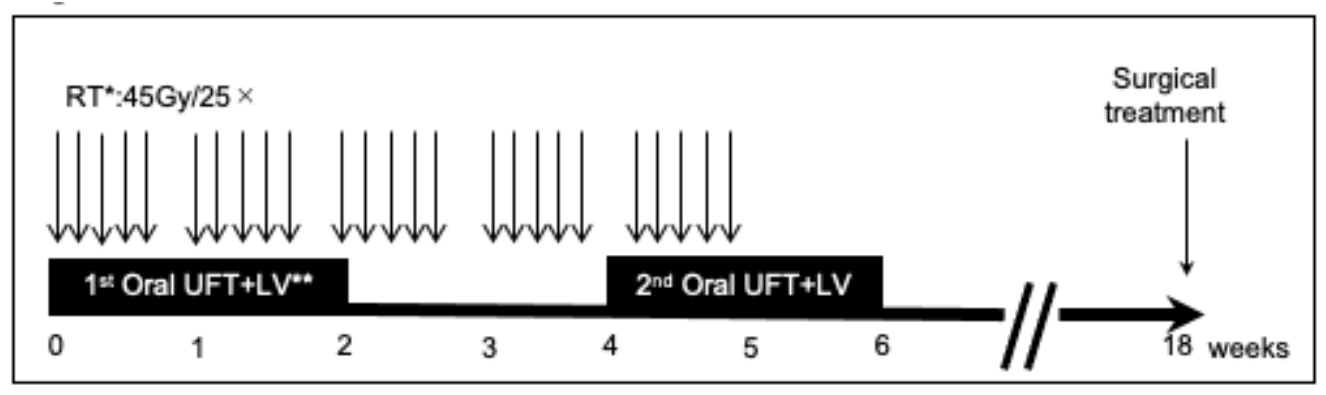

\section{Figure 1}

Protocol of preoperative CRT The oral UFT plus LV regimen consisted of 2 cycles, every 3 weeks. The dose of UFT was $300 \mathrm{mg} / \mathrm{m} 2 /$ day, and the dose of LV was $75 \mathrm{mg} /$ day on days $1-14$. Chemotherapy was intermittently repeated every 28 days, followed by a 2-week break. RT consisted of 45 Gy delivered in fractions of $1.8 \mathrm{~Gy} /$ day on weekdays for 5 consecutive weeks concomitantly with chemotherapy. 

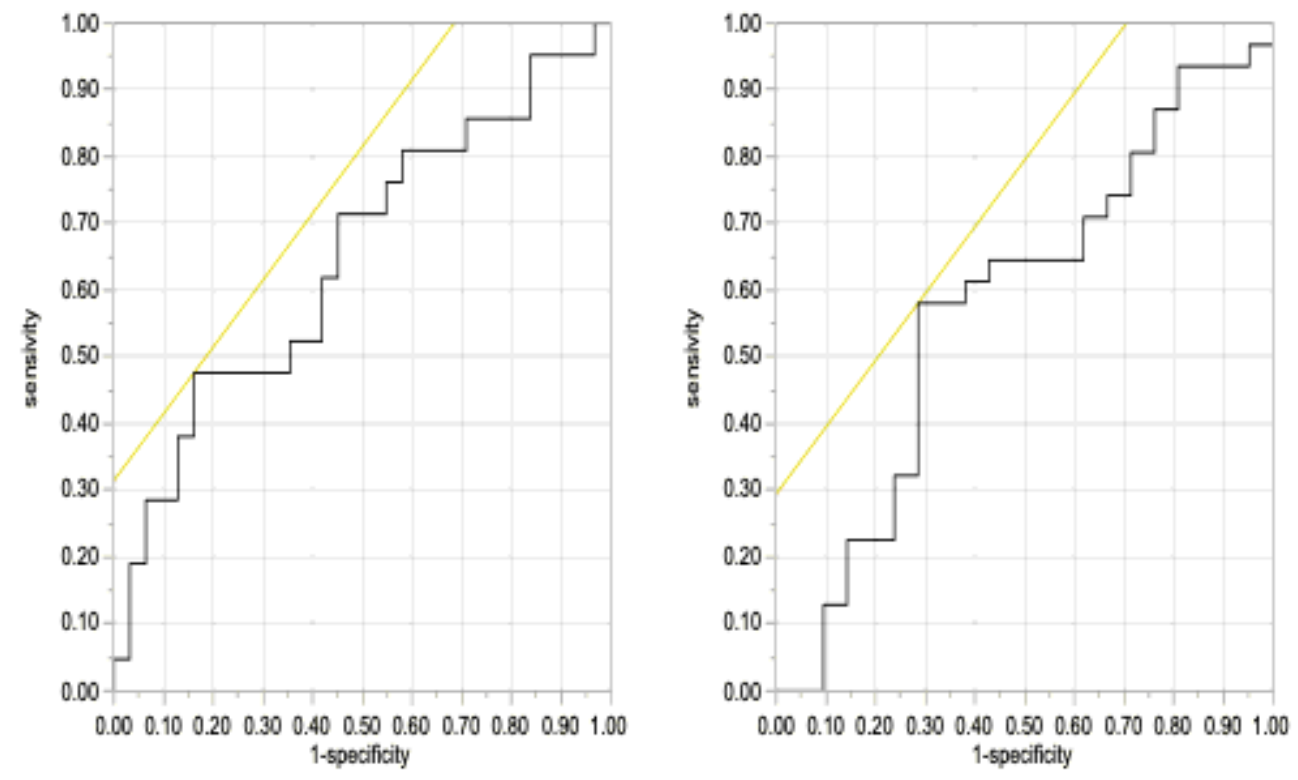

\section{Figure 2}

ROC curve for pre- and post-CRT NLR ROC curve to determine cut-off values for pre-CRT NLR. On the basis of ROC curves, cut-off values with the best discriminatory power for NLR was 3.20). AUC:0.648 Same as for post-CRT NLR. On the basis of ROC curves, cut-off values with the best discriminatory power for NLR was 4.38). AUC:0.579

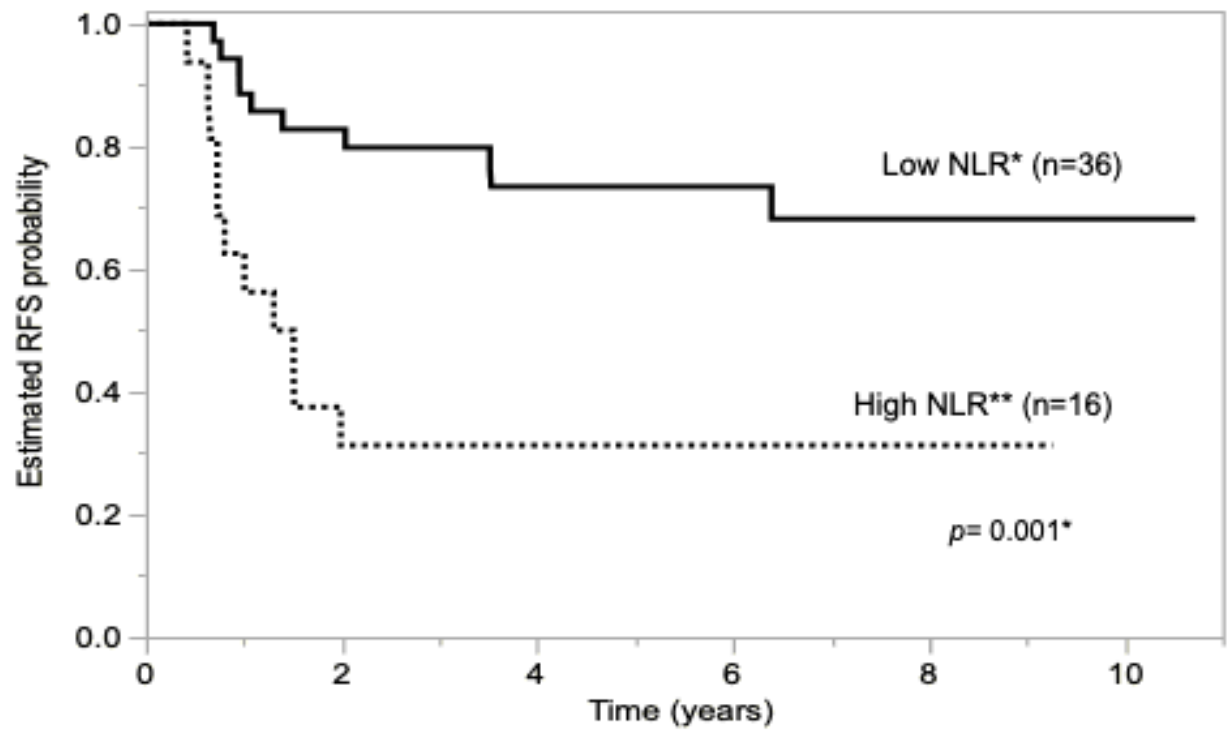

Figure 3

RFS according to NLR Recurrence-free survival according to pre-CRT NLR using Kaplan-Meier analysis. Group with pre-CRT $\geq 3.20$ showed lower 5-year RFS (31.3vs. $73.4 \%, p=0.01$ ). *Low NLR: Pre-CRT NLR<3.20 **High NLR: Pre-CRT NLR $\geq 3.20$. 


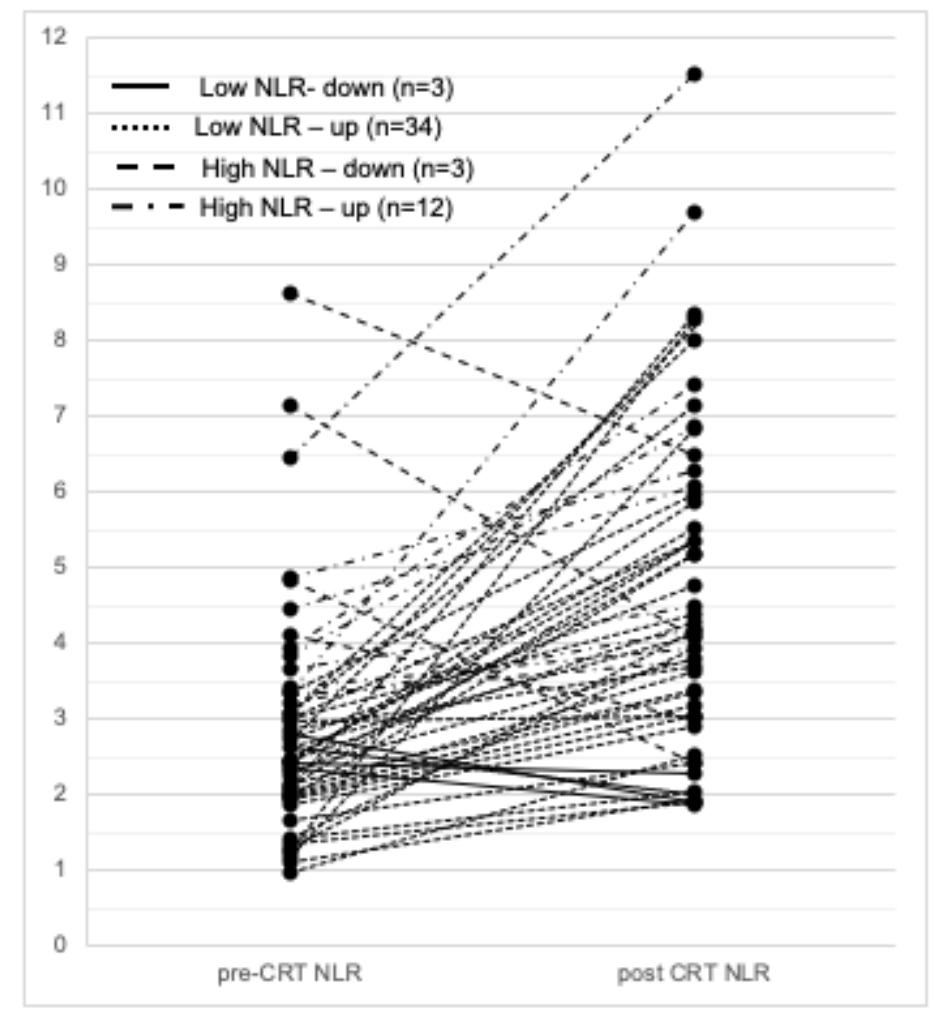

\section{Figure 4}

Comparing the increase and decrease in NLR value between pre- and post-CRT. Comparing the increase and decrease in NLR value between pre-CRT and post-CRT among these four groups, the four groups of “Low NLR-down”, “Low NLR-up”, "High NLR-down”, "High NLR-up”. 


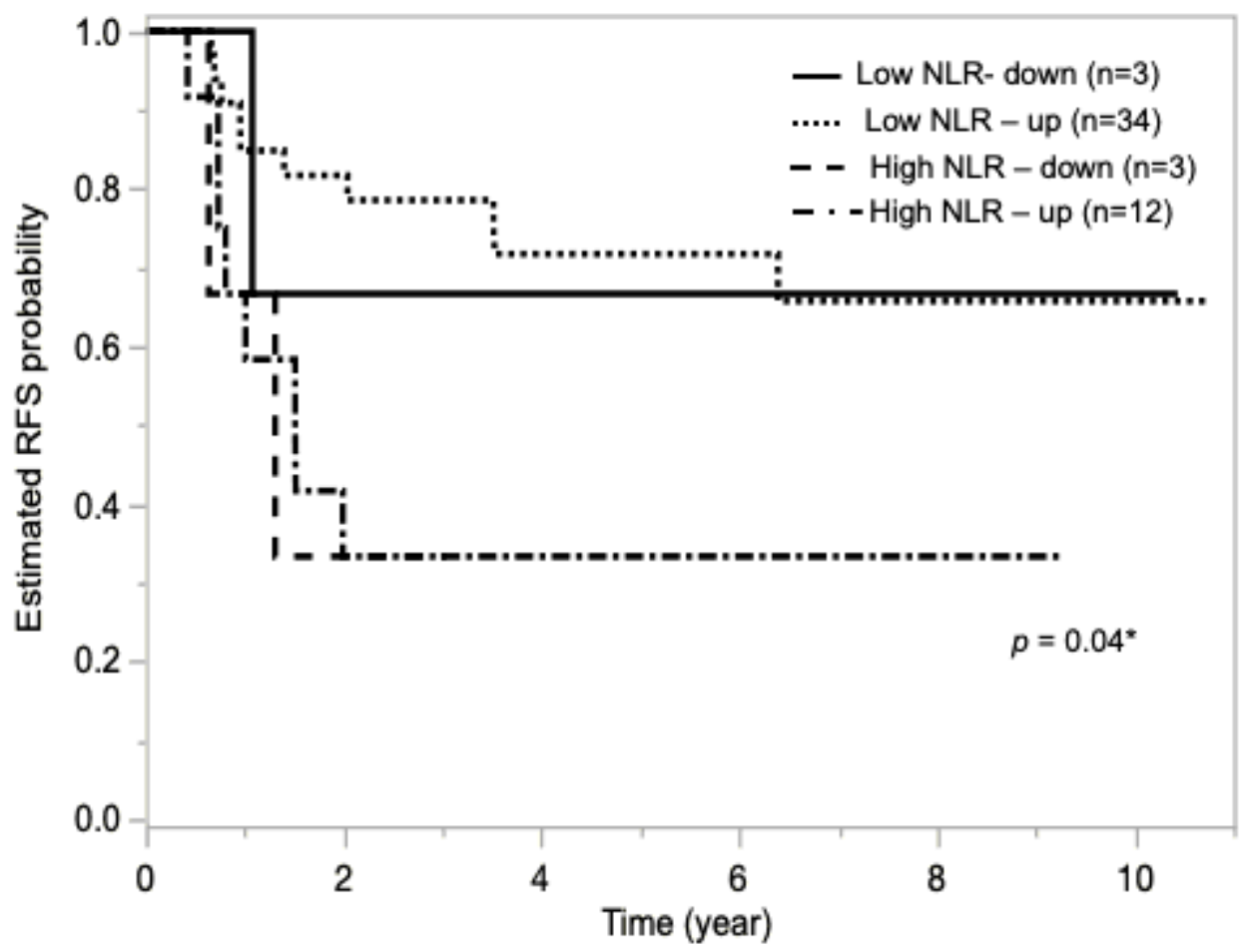

Figure 5

RFS according to fluctuation of pre- and post- NLR. RFS according to these four groups was assessed using Kaplan-Meier analysis. In particular, both the "High NLR-up" and "High NLR-down" group showed a lower 5-year RFS ( $p=0.04)$.

\section{Supplementary Files}

This is a list of supplementary files associated with this preprint. Click to download.

- Table1.tiff

- Table21.tiff

- Table22.tiff

- Table3.tiff 